\title{
Quantum relaxation in random magnets
}

\author{
J. Tejada and X. X. Zhang \\ Facultat de Física, Universitat de Barcelona, Diagonal 647, E-08028 Barcelona, Spain \\ E. M. Chudnovsky \\ Physics Department, Lehman College, City University of New York, Bedford Boulevard West, Bronx, New York 10468-1589
}

(Received 27 July 1992; revised manuscript received 30 November 1992)

\begin{abstract}
We present a comprehensive study of the low-temperature magnetic relaxation in random magnets. The first part of the paper contains theoretical analysis of the expected features of the relaxation, based upon current theories of quantum tunneling of magnetization. Models of tunneling, dissipation, the crossover from the thermal to the quantum regime, and the effect of barrier distribution on the relaxation rate are discussed. It is argued that relaxation-type experiments are ideally suited for the observation of magnetic tunneling, since they automatically provide the condition of very low barriers. The second part of the paper contains experimental results on transition-metal-rare-earth amorphous magnets. Structural and magnetic characterization of materials is presented. The temperature and field dependence of the magnetic relaxation is studied. Our key observation is a nonthermal character of the relaxation below a few kelvin. The observed features are in agreement with theoretical suggestions on quantum tunneling of magnetization.
\end{abstract}

\section{INTRODUCTION}

There is now a considerable interest devoted to the phenomena associated with quantum tunneling of magnetization in solids. ${ }^{1-6}$ Similar to quantum crystals, where quantum effects come to life because of the small mass of helium atoms, the magnetization $\mathbf{M}(\mathbf{r})$ was shown to be "light" enough to display quantum behavior in magnetically ordered systems.

Magnetic order in ferromagnets is created by the strong exchange interaction which aligns spins together. The orientation of the resulting $\mathbf{M}$ is determined by the energy of magnetic anisotropy, $E_{\text {an }}\left(M_{x}, M_{y}, M_{z}\right)$. In the absence of the anisotropy, any component of $\mathbf{M}$ conserves exactly since $\widehat{\mathbf{M}}$ commutes with the exchange Hamiltonian. In the presence of the anisotropy, however, the commutation of $\hat{\mathbf{M}}$ with the total Hamiltonian is, in general, violated. This is a simple reflection of the fact that the anisotropy is due to spin-orbit interactions. The magnetic anisotropy plays, therefore, a double role. On one hand, it orients $\mathbf{M}$ along some direction in a solid. On the other hand, it allows $\mathbf{M}$ to tunnel between different directions. $^{7}$

It has been demonstrated (see Sec. II) that there exists a characteristic (crossover) temperature $T_{c}$, below which the escape of $\mathbf{M}(\mathbf{r})$ from metastable configurations is dominated by quantum underbarrier transitions, not by thermal overbarrier activation. Although the exact value of $T_{c}$ depends on the shape of the barrier, the typical scale of $T_{c}$ is quite universally given by $\mu_{B}\left(H_{\|} H_{\perp}\right)^{1 / 2}$, where $H_{\|}$is the easy axis anisotropy field and $H_{\perp}$ is the field responsible for the noncommutation of $M_{\|}$with the Hamiltonian. For typical values of the magnetic anisotropy this corresponds to the temperature range $T_{c} \sim 0,1-10 \mathrm{~K}$. This prediction may seem to be inconsistent with observations. Indeed, magnetic materials have been investigated in that temperature range for a long time, and quantum behavior of the magnetization has not been widely noticed. There may be a simple explanation to that. The necessity of low temperature originates from the requirement $T<T_{c}$. In addition to that requirement, the rate of the escape must be sufficiently large to actually observe the transitions. This translates into the temperature-independent requirement of lowenergy barriers. The rate of thermal transitions is determined by the Boltzmann factor, $\Gamma_{T} \sim v \exp \left(-U / k_{B} T\right)$, where $U$ is the barrier height, $v$ is the attempt frequency. At $T \sim T_{c}$ the transition probability must begin to depart from this law, tending to the temperature-independent quantum transition rate, $\Gamma_{Q} \sim v \exp \left(-U / k_{B} T_{c}\right)$ at $T<T_{c}$. The prefactor $v$ is usually of the order of the ferromagnetic resonance (FMR) frequency, $v \sim 10^{10}-10^{11}$ $\mathrm{s}^{-1}$. Thus at $k_{B} T<</ 30$ thermal transitions are frozen out. Quantum transitions will reveal themselves on the time scale of the experiment, if the following condition is satisfied:

$$
k_{B} T<U / 30 \leq k_{B} T_{c} .
$$

There are two kinds of barriers in magnetic systems: intrinsic barriers due to magnetic anisotropy and barriers due to the pinning of domain walls by defects. Both are responsible for the pronounced metastability of magnetic materials, known as hysteresis phenomena. Anisotropy barriers are typically of the order of $H_{\|} M_{0} V$, while pinning barriers are of the order of $H_{c} M_{0} V$, where $M_{0}$ is the magnetic moment of the unit volume, $H_{c}$ is the coercive field, and $V$ is the volume involved in the tunneling process. These will produce the WKB exponent $U / k_{B} T_{c}$ of the order of $\left(H_{\|} / H_{\perp}\right)^{1 / 2} J$ for anisotropy barriers and of the order of $\left[H_{c} /\left(H_{\|} H_{\perp}\right)^{1 / 2}\right] J$ for pinning barriers, $J \sim M_{0} V / \mu_{B}$ being the total tunneling spin. Correspondingly, tunneling of a large spin $J \gg 30$ may occur only at 
$H_{\|} \ll<H_{\perp}$ or in materials with low coercitivity. To increase the total tunneling magnetic moment, one may think about the possibility to decrease the barriers by applying the magnetic field. A sufficiently large field will remove the barriers and establish a reversible magnetic behavior. This means that the problem of reducing the barriers essentially becomes the problem of the accuracy with which one can control the magnetic field.

It becomes clear from the above consideration that preparation of the system with small barriers is the most difficult part of the experiment on macroscopic (or mesoscopic) tunneling of the magnetization. The main purpose of this paper is to demonstrate, that, in fact, nature very often prepares for us magnetic systems which are ideally suited for investigation of tunneling effects.

Consider a magnetic system with high metastability and broad distribution of energy barriers. It may be a system of interacting single-domain particles, a highly defective ferromagnetic crystal, a random magnet, etc. As one applies the magnetic field, the magnetic moment of such a system typically has a two-step evolution. In the first, rapid stage, it changes due to the rotation of the local magnetization in areas where the barriers are removed by the field. As the magnetization of the system changes, so does the internal field inside the magnet. Eventually, the evolution of the system gets stuck in the state where the barriers just begin to develop. The later, slow phase of the evolution is due to thermal or quantum transitions in the presence of the barriers. Thus, starting from zero barriers, the relaxing system automatically arrives at the barrier heights for which the lifetime of metastable states corresponds to the actual time scale of the experiment. This situation is well known in the theory of type-II superconductors, ${ }^{8-11}$ where the slow relaxing phase starts from the Bean critical state. ${ }^{12}$ It can be easily understood in terms of the friction-dominated motion of a block down the incline with a decreasing slope. The block stops when the moving force due to gravity is balanced by the friction. The following slow motion is possible if somebody is shaking the incline (the analog of temperature).

For thermally activated processes, the rate of the slow relaxation discussed above must decrease as temperature goes down. Independence of this rate of temperature, below some temperature, is the vestige of quantum tunneling.

In this paper we will report observation of such behavior in random magnets and will attempt to make a link between theory and experiment.

The paper is organized as follows. In Sec. II we review the existing models of quantum tunneling of magnetization. The effect of dissipation on the tunneling rate and expected features of the crossover from thermal to quantum regime are discussed. Different approaches to the understanding of $\log (t)$ relaxation are considered. Section III contains experimental results on $\mathrm{Fe}-\mathrm{Sm}, \mathrm{Fe}-\mathrm{Tb}$, and Co-Sm amorphous magnets. Structural and magnetic characterization of these materials is presented. Our key observation concerns the low-temperature magnetic relaxation. In Sec. IV the experimental results are discussed in terms of quantum tunneling of magnetization.

\section{THEORY}

\section{A. Models of tunneling of magnetization}

A few models of tunneling have recently been suggested. The most simple situation corresponds to a singledomain ferromagnetic particle. ${ }^{1,2}$ Let us assume that the magnetic moment of the particle points along the $Z$ axis and the field is applied in the opposite direction. The stable magnetic state of the particle corresponds to $\mathbf{M}$ directed along $\mathbf{H}$. To rotate an individual atomic magnetic moment with respect to others costs the exchange energy $\epsilon_{\text {ex }}$ per atom. To coherently rotate the total magnetic moment of the particles out of the anisotropy axis costs $\epsilon_{\text {an }} N$, where $N$ is the total number of magnetic atoms. Thus, at $N<\epsilon_{\mathrm{ex}} / \epsilon_{\mathrm{an}} \sim 10^{3}-10^{5}$ it is energetically favorable to develop a uniform rotation of $\mathbf{M}$ towards the minimal energy state. The Hamiltonian of the model that possesses tunneling must not commute with the component of $\mathbf{M}$ that corresponds to its equilibrium orientation. Consequently, in the case of the uniaxial anisotropy, tunneling may only be due to the magnetic field off the easy axis. To ensure a significant tunneling rate such a field must be comparable to the anisotropy field.

In our experiments (see Sec. III) the applied field will always be less than the anisotropy field, so we are primarily interested in situations where tunneling is due to magnetic anisotropy itself. The minimal nonuniaxial model of that kind which contains tunneling corresponds to the orthogonal symmetry, with the energy density $E_{\text {an }}=-k_{\|} M_{z}^{2}+k_{\perp} M_{y}^{2}-\mathbf{M H}\left(k_{\|}, k_{\perp}>0\right)$. This model contains all the basic features of more complicated situations. In a spherical coordinate system it is equivalent to

$$
E_{\text {an }}=\left(K_{\|}+K_{\perp} \sin ^{2} \phi\right) \sin ^{2} \theta-M H(1-\cos \theta),
$$

where $\theta(r)$ and $\phi(r)$ are spherical coordinates of a fixed length vector $\mathbf{M}(\mathbf{r})$. For $H<H_{\|}=2 k_{\|} / M_{0}$, there are two local energy minima: $\theta=0$ and $\pi$; the maximum (at $\phi=0$ ) corresponds to $\cos \theta_{1}=H / H_{\|}$, and determines the barrier between the minima (Fig. 1). The height of the barrier is $U=K_{\|} V \varepsilon^{2}$, where $V$ is the volume of the particle, $\varepsilon=1-H / H_{\|}$. Notice that the transverse anisotropy $k_{\perp}$ is responsible for quantum transitions between the minima; if $k_{\perp}=0, M_{z}$ commutes with $E_{\text {an }}$. The WKB exponent of the transition is given by the Euclidean action

$$
S_{E} / \hbar=-i V / \hbar \int d t\left[\left(M_{0} / \gamma\right) \dot{\phi} \cos \theta-E_{\mathrm{an}}(\theta, \phi)\right]
$$

evaluated along the instanton trajectory leading $M$ out of the metastable state. This is equivalent to solving the Landau-Lifshitz-Gilbert equation

$$
\partial \mathbf{M} / \partial t=-\gamma \mathbf{M} \times \delta E_{\text {an }} / \delta \mathbf{M}
$$

in imaginary time. Surprisingly, it has been quite recently noticed that while the real-time solutions of Eq. (3) describe phenomena such as FMR, spin waves, and propagation of domain walls, the imaginary-time solutions correspond to tunneling events. ${ }^{1}$ For $E_{\text {an }}$ given by Eq. (1) one obtains that the quantum underbarrier transition probability $P$ is 


$$
P \propto \exp \left(-U / k_{B} T_{c}\right),
$$

where $^{2}$

$$
k_{B} T_{c}=\frac{3}{4} \mu_{B}\left(H_{\|} H_{\perp}\right)^{1 / 2} \varepsilon^{1 / 2} .
$$

In the case of the uniaxial anisotropy of the particle, $k_{\|}$, the transitions between the energy minima can be induced by the field applied at some angle to the anisotropy axis. If the field is perpendicular to the axis, the transition probability at small $\varepsilon=1-H / H_{\|}$is given by Eq. (4) with $^{2} U=K_{\|} V \varepsilon^{2}, k_{B} T_{c}=\frac{1}{4} \mu_{B} H_{\|} \varepsilon^{1 / 2}$.

A similar approach has been developed for the problem of quantum nucleation in a bulk ferromagnet. ${ }^{2}$ In this case one must add the exchange energy $\left(A / M_{0}^{2}\right)\left(\partial_{i} \mathbf{M}\right)^{2}$ to the anisotropy energy and look for nonuniform imaginary-time solutions of Eq. (3). A nice example is quantum nucleation of a cylindrical bubble in a ferromagnetic film (Fig. 2). The probability of that process is given by Eq. (4) with $U=23.3 K_{\|} h \delta^{2} \varepsilon$ and $k_{B} T_{c}$ $=1.24 \mu_{B}\left(H_{\|} H_{1}\right)^{1 / 2} \varepsilon^{1 / 2}$ for orthogonal magnetic anisotropy. Here $h$ is the thickness of the film and $\delta=\left(A / K_{\|}\right)^{1 / 2}$ is the thickness of the domain wall.

Until now we considered only tunneling through intrinsic barriers due to the magnetic anisotropy. Another class of problems is represented by tunneling of domain walls through barriers created by pinning defects. ${ }^{13,14}$ In this problem the wall is described by the equation of the surface $Z=Z(x, y, t)$. The WKB exponent for the tunneling is given by the external Euclidean action of the wall

$$
\begin{aligned}
& S_{E}=\left(-\sigma \delta^{2} / \omega\right) \int d^{3} x(\left\{1+[\nabla \bar{z}(\mathbf{x})]^{2}\right\}^{1 / 2} \\
&\left.+u\left(x_{1}, x_{2}, \bar{z}\right)-h \bar{z}(\mathbf{x})\right),
\end{aligned}
$$

where $\sigma$ is the energy per unit area of the wall, $\omega=v_{0} / \delta$, $v_{0}$ is the limiting velocity of the wall, $x_{1}=x / \delta, x_{2}=y / \delta$, $x_{3}=i \omega t, \bar{z}=z / \delta, u=U(x, y, z) / \sigma$ is the pinning potential, $h=2 M_{0} H \delta / \sigma$.

Analysis of this problem for different situations shows that typically the WKB exponent for the tunneling is of the order of ${ }^{14}$

$$
B \sim\left(\mu_{B} H_{\mathrm{an}} / \hbar \omega\right)\left(h_{c} \varepsilon\right)^{5 / 4} J,
$$

where $h_{c}=H_{c} / H_{\mathrm{an}}, H_{c}$ is the coercive field, and

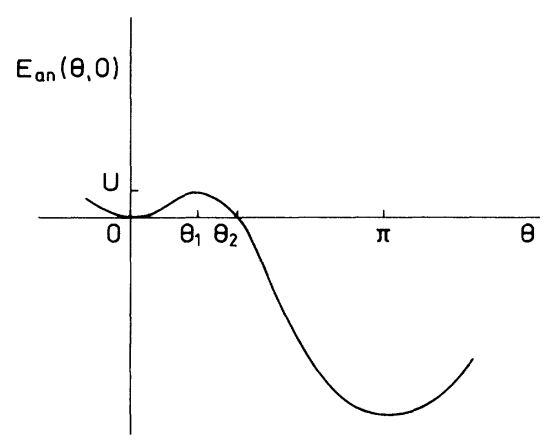

FIG. 1. The anisotropy energy $E_{\text {an }}(\theta, \Phi=0)$.

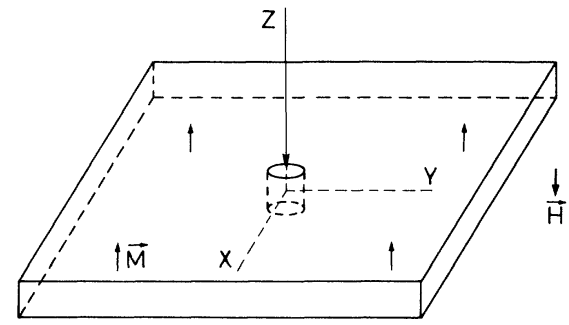

FIG. 2. Nucleation of a cylindrical bubble in a ferromagnetic film.

$\varepsilon=1-H / H_{c}$. In the materials with low coercitivity, the total tunneling spin can be quite large, $J \sim 10^{6}$. The crossover temperature for the domain wall tunneling is ${ }^{14}$

$$
k_{B} T_{c} \sim \hbar \omega\left(h_{c} \varepsilon\right)^{1 / 4} .
$$

Due to the weak dependence on $h_{c}$ and $\varepsilon$, it can be rather high, $T_{c} \sim 1 \mathrm{~K}$, even at $h_{c}, \varepsilon \ll 1$.

All the above formulas have been derived for some ideal geometrics of tunneling and, thus, cannot be used for a precise analysis of experimental situations if these ideal conditions are not satisfied. Nevertheless, they provide experimentalists with a rough estimate of the expected tunneling rate and the crossover temperature based upon macroscopic characteristics of magnetic materials. In particular, one may expect that a higher magnetic anisotropy will allow one to observe tunneling at a higher temperature. The number of tunneling spins can also be estimated based upon the height of the barrier.

\section{B. Dissipation}

When studying the tunneling of a macroscopic object, as the magnetization $\mathbf{M}(\mathbf{r}, t)$, one should be concerned about its interaction with microscopic degrees of freedom, that is, the dissipative environment. The importance of this interaction immediately becomes clear, if one notices that a baseball in a potential well will not move either classically or quantum mechanically even in the limit of a flat horizontal surface when the barrier becomes zero. The reason is friction. A similar argument, in principle, may apply to the tunneling of $\mathbf{M}$.

For couplings to the environment which are linear in the environmental variables, the general answer to the question of whether dissipation is important or not was given by Caldeira and Leggett. ${ }^{15}$ They found that most serious effects come from Ohmic coupling, which in classical terms corresponds, e.g., to the motion of the particle of mass $m$ in the potential $U(x)$, satisfying

$$
\ddot{x}+v_{d} \dot{x}=\frac{-1}{m}\left(\frac{\partial U}{\partial x}\right) .
$$

The remarkable result of Caldeira and Leggett is that the effect of such coupling on the WKB exponent $B$ can be presented in terms of the dissipation constant $v_{d}$,

$$
B \sim B_{0}\left(1+h v_{d} / k_{B} T_{c}\right),
$$


where $B_{0}$ is the WKB exponent in the absence of dissipation.

For tunneling of the magnetization, dissipative interactions include couplings to conducting electrons, phonons, and magnons. Conducting electrons are important in metals where time-dependent $\mathbf{M}$ induces eddy currents. Interaction of $\mathbf{M}$ with the conducting electrons is Ohmic in nature. It has been argued ${ }^{14}$ that the contribution of this interaction to the tunneling rate can be approximated by Eq. (10) with $v_{d}$ being the width of the ferromagnetic resonance. Typically this contribution is small.

Interaction with phonons corresponds to the $u_{i k} M_{i} M_{k}$ term in the Hamiltonian of the system, where $u_{i k}$ is the strain tensor. It is linear but non-Ohmic. The relative contribution of this interaction to the WKB exponent has been estimated ${ }^{16}$ as $10^{-5}$. The contribution of magnons is more difficult to elaborate since it is nonlinear and non-Ohmic. Theoretical study of the magnon contribution $^{13,14}$ shows that it cannot be large, but can, in principle, be of the order of one at $T \sim T_{c}$, and rapidly goes to zero as temperature is lowered. The relative contribution of the dissipation on the domain wall tunneling has been estimated as ${ }^{14}$

$$
\left(\beta_{a} \gamma \delta / \mu_{H}\right)\left(h_{c} \varepsilon\right)^{-1 / 4},
$$

where $\beta_{a}$ is a factor determined by the structure of the magnetic anisotropy (for, e.g., a rhombic crystal, $\left.\beta_{a}=\left[\left(1+K_{\perp} / K_{\|}\right)^{1 / 2}-1\right]\right), \gamma$ is the gyromagnetic ratio, $\mu_{H}$ is the mobility of the wall with respect to the magnetic field, $v=\mu_{H} H$. This contribution can, in principle, be noticeable in conducting materials with low mobility of domain walls.

The general conclusion of studies on the effect of dissipation on magnetic tunneling is that it cannot significantly affect the results obtained without dissipation, but may, in principle, be noticeable in the case of very low barriers.

\section{Crossover from quantum to thermal regime}

As has been discussed in the Introduction, the escape of the magnetization from a metastable state must be governed by the Boltzmann exponent, $\exp \left(-U / k_{B} T\right)$, at high temperature, and must be given by the temperature-independent WKB exponent, $\exp (-B)$, at $T \rightarrow 0$. The crossover between two regimes occurs at $k_{B} T \sim U / B$. A common mistake is to think about thermal and quantum transitions as independent processes which contribute additively to the total escape rate. This is not true because the total escape rate is formed by interfering channels of the transition. It may be evaluated within a nice universal approach based upon functional integration. Since the temperature dependence of the escape rate is the key to the understanding of our experimental results, we will give here a brief sketch of the theory. ${ }^{1,17-19}$

Although the tunneling of the magnetization may be associated with different processes, there is no reason to believe that the general features of the crossover for these processes are essentially different from the thermal-toquantum crossover in the problem of a particle escaping from a metastable state. In fact, it has been demonstrated that the problem of magnetic moment tunneling in a single-domain particle can be explicitly mapped onto the tunneling problem in mechanics. ${ }^{20}$ Consider a particle of mass $M$ in a metastable state formed by the potential $V(q)$ (Fig. 3). The probability of the escapes, $\Gamma$, is assumed to be exponentially small, providing that the particle is in a thermodynamic equilibrium with the environment. It is given by the functional integral

$$
\Gamma \sim \int D\{q(\tau)\} \exp (-1 / \hbar \oint d \tau L[q(\tau)]),
$$

where $L[q(\tau)]$ is the imaginary-time ( $\tau=i t$ ) classical Lagrangian of the system, the functional integration is performed over $q(\tau)$ trajectories which are periodic in $\tau$ with the period $\tau_{p}=\hbar / k_{B} T$, and the integral in the exponent is taken over the period $\tau_{p}$. In a semiclassical limit, the decay rate, with an exponential accuracy, is

$$
\Gamma \propto \exp \left(-S_{E} / \hbar\right),
$$

where $S_{E}$ is the minimal Euclidean action evaluated along the $q(\tau)$ trajectory with $\tau_{p}=\hbar / k_{B} T$ that satisfies $M \ddot{q}=d V / d q$. Periodic solutions of this equation belong to two classes: a constant, $q=q_{0}$, and $q(\tau)$ satisfying

$$
\frac{1}{2} M \dot{q}^{2}=V(q)-E\left(\tau_{p}\right) .
$$

The first solution, $q=q_{0}$, corresponds to the particle at rest at the bottom of the inverted potential (Fig. 3). The second solution, $q(\tau)$ given by Eq. (14), corresponds to the periodic motion of the particle in the inverted potential between $q_{1}(E)$ and $q_{2}(E)$ (Fig. 3), with $E$ determined by the condition that the period of the motion equals $\hbar / k_{B} T$.

For $q=q_{0}$ one obtains

$S_{E}=\oint d \tau\left[\frac{1}{2} M \dot{q}^{2}+V(q)\right]=V\left(q_{0}\right) \tau_{p}=\hbar U / k_{B} T$,

which gives the Boltzmann formula after substitution

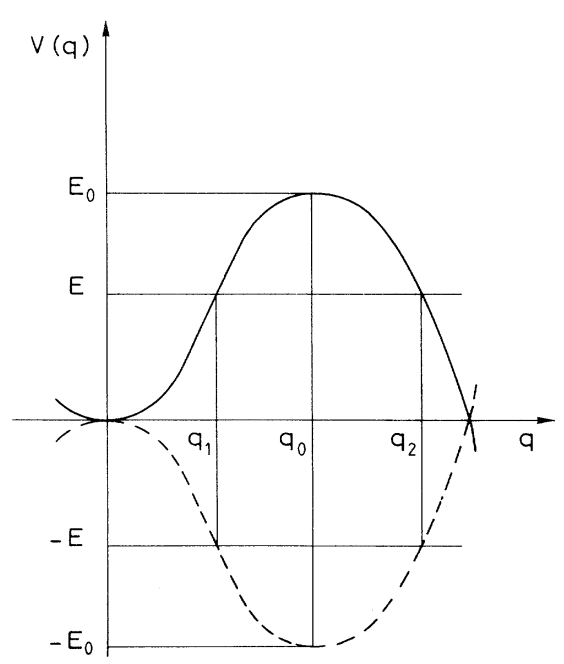

FIG. 3. The barrier $V(q)$ (solid line) and the inverted barrier $-V(q)$ (dashed line). 
into Eq. (13). This solution must be used at $T>T_{c}$ where $T_{c}$ is the temperature at which periodic, with $\tau_{p}=\hbar / k_{B} T$, solutions of Eq. (14) no longer exist. For the class of potentials for which $\tau_{p}$ is monotonically decreasing with $E, k_{B} T_{c}=\hbar \omega_{0} / 2 \pi$, where $\omega_{0}$ is the frequency of small oscillations near the bottom of the inverted potential (Fig. 3). At $T<T_{c}\left(\tau_{p}>2 \pi / \omega_{0}\right)$ the Euclidean action is minimized by periodic solutions of Eq. (14). The escape rate at any temperature can be written as

$$
\Gamma=v(T) \exp \left[-U / k_{B} T^{*}(T)\right],
$$

with $T^{*}=T$ at $T>T_{c}$ and $T^{*}(T)>T$ at $T<T_{c}$. A more detailed analysis shows that the dependence of $T^{*}$ on $T$ is always of the form shown in Fig 4 .

A nontrivial consequence of the theory is that, in the steepest-descent approximation, the transition from thermal to quantum regime is rather sharp, with a wellestablished plateau in the $T^{*}(T)$ dependence. Recently, it has been demonstrated ${ }^{21}$ that, for potentials having a nonmonotonic $\tau_{p}(E)$ dependence, it can be even sharper, with $k_{B} T_{c}>\hbar \omega_{0} / 2 \pi$. The effect of the dissipation has been shown to smear the transition. ${ }^{22-24}$ The dependence $T^{*}(T)$ may, therefore, bring information on the relative contribution of the dissipation to the tunneling rate.

\section{Critical state and distribution of energy barriers}

To observe tunneling, an ideal experimental system would be a set of identical noninteracting single-domain particles frozen in a nonmagnetic matrix, in the presence of the magnetic field which aligns all magnetic moments in one direction. As the field is removed, the total magnetic moment of that system will decay with time according to

$$
M(t)=M(0) e^{-\Gamma t},
$$

with $\Gamma$ given by Eq. (16).

In practice, however, it is very difficult to prepare a system of noninteracting identical particles, or identical energy barriers. The barrier is proportional to the volume of the particle, or, more generally, to the volume $V$ involved in the tunneling process. Thus, at $T \rightarrow 0$, the

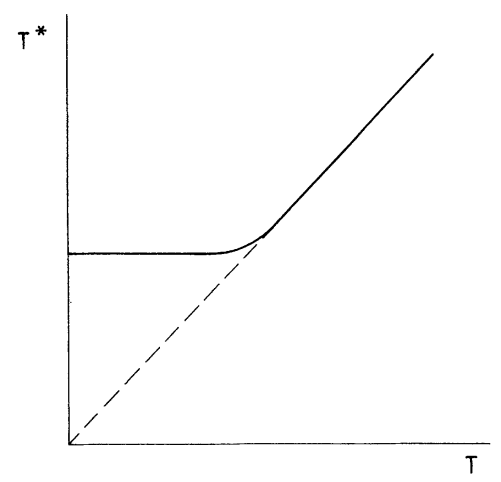

FIG. 4. $T^{*}(T)$ dependence. tunneling rate can be presented as

$$
\Gamma_{0}=v(0) \exp \left(-B_{0} r^{3} / r_{0}^{3}\right),
$$

where $r$ is the linear size of the tunneling volume and $r_{0}$ is the size of the volume corresponding to the WKB exponent $B_{0}$. As has been discussed in the Introduction, a typical value of $B_{0}$, dictated by the time of the experiment, is $B_{0} \sim 30$. Consequently, a size distribution within only $3 \%$ around $r_{0}$ will change the tunneling rate by the order of magnitude. This must be taken into account in all resonance experiments ${ }^{6}$ based upon the assumption of narrow size distribution.

Fortunately, however, even very complex magnetic materials can reveal a clear signature of quantum tunneling of magnetization in relaxation-type experiments. Consider a highly metastable magnet uniformly magnetized by the applied magnetic field. If the field is suddenly removed, the magnetization immediately drops to a finite value determined by the hysteresis curve. This is the magnetic state where the energy barriers just start to develop. It is similar to the Bean critical state ${ }^{12}$ in superconductors. In the latter case, the Lorentz force on flux lines is balanced by the local pinning force.

In magnets it may, e.g., be the balance between the pinning of domain walls and the magnetic force on the walls. The $\log (t)$ magnetic relaxation in the critical state, commonly observed in magnets ${ }^{4,5}$ and superconductors, ${ }^{9-11}$ can be explained within the Anderson-Kim model. ${ }^{25,26}$

At $\Gamma t<<1$ the time derivative of the magnetization is

$$
d M / d t=-M(0) \Gamma .
$$

Let us start with a pure thermal activation. Assuming that the barriers begin to develop at $M<M_{c}$ (Fig. 5), $\Gamma$ can be written as

$$
\Gamma=v \exp \left[-\left(\frac{U_{0}}{k_{B} T}\right)\left(1-\frac{M}{M_{c}}\right)\right] .
$$

Here $M_{c}$ is related to the coercive field by $H_{c}=N M_{c}$, where $N$ is the demagnetization factor. The solution of Eq. (19) is

$$
M(t)=M\left(t_{0}\right)\left[1-\frac{k_{B} T}{U_{0}} \ln \left(\frac{t}{t_{0}}\right)\right],
$$

where $M\left(t_{0}\right) \approx M_{c}$. It is easy to check that the condition $\Gamma t<1$ is equivalent to $k_{B} T \ll<U_{0}$.

One should notice that the model presented by Eq. (20) is very approximate. A complex system is expected to be in a critical state locally, that is, at any point. One should also expect that these local states are characterized by different energy barriers. This does not change Eq. (21) but suggests that $U_{0}$ must be understood as some average barrier. There is also a possibility that local energy barriers are proportional to $\left(1-M / M_{c}\right)^{p}$ where $p \neq 1$. This may change the power of $\ln (t)$ in Eq. (20). The statistical theory of the relaxation in a critical state is yet to be developed.

It was noticed long ago ${ }^{27,28}$ that a broad distribution of energy barriers may provide the $\log (t)$ relaxation even outside the critical state. Consider a system of nonin- 


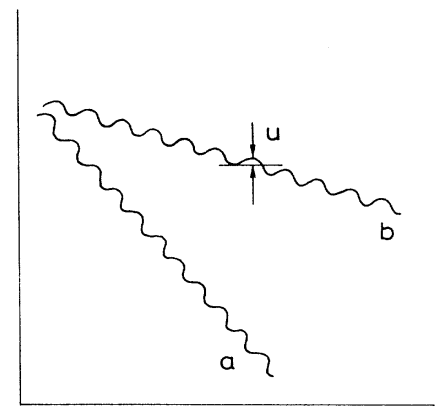

FIG. 5. Schematic representation of the potential relief. (a) Critical state (zero barriers). (b) Departure from the critical (finite barriers).

teracting single-domain particles or magnetic clusters of similar size but with a broad distribution of barrier heights. This may be due to, e.g., fluctuations in the anisotropy constant or distribution of easy axes with respect to the orientation of the applied field. In this case Eq. (17) must be replaced by

$$
M(t)=M(0) \int d U f(U) e^{-\Gamma t},
$$

where $\Gamma=v \exp \left(-U / k_{B} T\right), f(U)$ is the distribution function, $\int f(U) d U=1$. Time derivative of this equation may be written as

$$
\frac{1}{M(0)} \frac{d M}{d t}=-\frac{k_{B} T}{t} \int_{0}^{\infty} d x e^{-x} f\left(k_{B} T \ln (v t / x)\right),
$$

where we have substituted $x \equiv \Gamma t$. For a nonsingular distribution function, the main contribution to the integral (23) comes from $x \sim 1$, that is, from metastable states having a lifetime $\Gamma^{-1}$ comparable to the observation time $t$. For a typical relaxation experiment $t \sim 1-10^{4} \mathrm{~s}$, that is, $v t \sim 10^{11}-10^{15}$. At $x \sim 1$, this gives $\ln (v t)>\ln x$, so that Eq. (23) with good accuracy becomes

$$
\frac{1}{M(0)} \frac{d M}{d t}=-\frac{k_{B} T}{t} f\left(k_{B} T \ln (v t)\right) .
$$

Integration gives

$$
M(t)=M\left(t_{0}\right)\left[1-\int_{0}^{y(t)} d y^{\prime} f\left(y^{\prime}\right)\right],
$$

where $t_{0}=v^{-1} \sim 10^{-11} \mathrm{~s}, M(0)$ is replaced by $M\left(t_{0}\right)$ for a proper treatment of small times,

$$
y(t)=k_{B} T \ln (v t) .
$$

An important observation follows from Eqs. (25) and (26): $M$ depends on $t$ through the combination (26). This means, for example, that if one observes the $\log (t)$ magnetic relaxation due to the barrier distribution, the coefficient in front of $\log (t)$ must be proportional to $T$.

The commonly observed, linear in $\log (t)$ relaxation, can be easily understood from Eq. (25). Let $U_{0}$ be the average energy barrier which determines the blocking temperature, $k_{B} T_{B} \sim U_{0} / 30$ in static $M(T, H)$ measure- ments. At $T \ll T_{B}$ the integral in Eq. (25) can be approximated by the first term of the expansion in a series on $y / U_{0}$,

$$
M(t)=M\left(t_{0}\right)\left[1-k_{B} T f(0) \ln \left(t / t_{0}\right)\right],
$$

where $f(0) \sim U_{0}^{-1}, t_{0}$ is arbitrary. This formula describes the low-temperature relaxation due to transitions through small barriers, at the tail of the distribution. As for the critical state relaxation, it is assumed that the relaxing part of the magnetic moment is small compared to the total moment. One should expect this condition to become invalid at large $t$ for $T \sim T_{B}$.

One may be puzzled by the fact that such different models, the critical state model and the barrier distribution model, lead to the same result, Eqs. (21) and (27). The answer becomes clear if one notices that both models have one distinct feature: as the observation time is running, the system arrives at greater and greater barriers which are more difficult to overcome. This is a very natural feature of complex systems and the key to understanding the $\log (t)$ relaxation. Thus the relaxation proportional to $T \log (t)$ at $T_{c} \ll T<<T_{B}$ must be expected, and does occur, in a large variety of magnetic materials.

Let us now turn to the low-temperature relaxation where quantum effects are expected. Derivations of Eqs. (21) and (27), together with Eq. (16), simply suggest that we have to replace $T$ in Eqs. (21) and (27) by $T^{*}(T)$. At large $T$ this function has a universal $T$ dependence, $T^{*} \rightarrow T$. At small $T$, however, it depends on the shape of the energy barrier (Sec. II C). In the case of the distribution of barriers, the relaxation law becomes

$$
M(t)=M\left(t_{0}\right)\left[1-S(T) \ln \left(t / t_{0}\right)\right],
$$

where $S(T)$ is given by

$$
S(T)=\left[k_{B}\left\langle T^{*}(T)\right\rangle\right] /\langle U\rangle .
$$

$\left\langle T^{*}(T)\right\rangle$ means averaging over the barriers of different shape. As has been discussed in Secs. II A and II C, $T^{*}(T)$ does not depend on the extensive parameters such as, e.g., the tunneling volume. Even for a broad distribution of tunneling volumes, one should expect a relatively narrow distribution in $T^{*}(T)$ at low temperature. This suggests that $S(T)$, commonly called the magnetic viscosity, must have a universal behavior similar to that shown in Fig. 4. The existence of the plateau in $S(T)$ at low temperature should be interpreted as the evidence of quantum tunneling of magnetization.

Our final remark concerns possible interaction between tunneling clusters. The critical state arguments are equally applied to that case, since they do not specify the nature of the energy barriers. The arguments used in the model of barrier distribution will change, however, because Eq. (22) is explicitly based upon the assumption that tunneling clusters relax independently. This may change the conclusion about the proportionality of $S(T)$ to temperature at large $T$. However, the conclusion about the plateau in $S(T)$ dependence at low temperature due to quantum effects should remain unchanged. 


\section{EXPERIMENT}

\section{A. Samples and equipment}

The materials studied are compositionally modulated thin films of rare-earth (RE)-transition-metal (TM) alloys. Depending on the thickness of layers, on the rate of the deposition, and on the temperature of the substrate, RE-TM multilayered systems exhibit a large variety of magnetic properties. When the two consecutive TM and RE are very thin, a single TM-RE ferromagnetic amorphous layer is formed due to the interdiffusion of metals. This was the case for our samples.

Our systems were prepared by using two electron beam evaporators and in a high-vacuum chamber. The pressure during the evaporation of metals was maintained at $5 \times 10^{-7}$ torr. The evaporation rate controlled by the quartz crystal oscillator was $0.5 \AA / \mathrm{s}$. A very thin kapton foil was used as a substrate. Before evaporating RE and TM metals, a $100-\AA$ buffer of a nonmagnetic metal $(\mathrm{Cu}$ or $\mathrm{Ag})$ has been deposited onto kapton. The substrate was kept at $260^{\circ} \mathrm{C}$ during the evaporation. The composition of the samples, the thickness, and other parameters of the layers are summarized in Table $I$.

The modulation in the composition was checked by the scanning tunneling microscope and by the low angle $\mathrm{x}$ ray diffraction. The high angle $\mathrm{x}$-ray diffraction data show only peaks corresponding to $\mathrm{Cu}(111)$ and crystalline silver. The final composition of the intermetallic layers was deduced from the scanning electron probe analysis. For the two samples containing iron, we also recorded Mössbauer spectra between $4 \mathrm{~K}$ and room temperature. The Mössbauer data confirmed the random magnetic structure of the films.

Magnetic measurements were performed by using a superconducting quantum interference device (SQUID) magnetometer. During the relaxation experiments, the data were corrected to take into account the spurious drift of the SQUID baseline. The applied magnetic field in the relaxation studies was produced by using a power supply which had a record constancy in the generated current. The accuracy in the field measurement was better than 0.1 Oe. The applied field was calibrated by using a pure $\mathrm{Pb}$ sample in the superconducting state. The constancy of the applied field was checked by measuring the signal from a pure paramagnetic sample during two hours at different temperatures.
The following procedure was used in all relaxation measurements. The sample was cooled from room temperature to a well-defined final temperature in the presence of a 100-Oe field. Then the field was rapidly changed from 100 Oe through zero to -100 Oe. The first measurement of the magnetization was taken four seconds after the change of the field. The variation of the magnetization with time was then followed during the time period up to a few hours. The time interval between two consecutive $M(t)$ points was $4 \mathrm{~s}$. The applied field during the relaxation experiments can, in principle, take any value $H$ verifying $0 \leq H<H_{\|}$. We applied a field $H=-100$ Oe after the field-cooled process in order to detect better the relaxation phenomena.

\section{B. Static properties}

\section{High field measurements}

Hysteresis measurements at different temperatures were performed for all the samples in order to get the variation with temperature of the coercive field $H_{c}$, the remanence magnetization $M_{r}$, the saturation magnetization $M_{s}$, and the anisotropy field $H_{\text {an }}$. These measurements were done for the applied field parallel and perpendicular to the film plane. The total magnetization of the samples has been found to lie in the plane. In Fig. 6 we show the hysteresis data for the FeTb sample at $5 \mathrm{~K}$. Figure 7 shows the variation of the coercive field with the temperature for the FeSm sample.

RE-TM random magnets are characterized by strong ferromagnetic exchange and random magnetic anisotropy. Such systems have been successfully described within the correlated spin-glass model. ${ }^{29-32}$ According to this model, the atomic magnetic moments are ferromagnetically correlated on a small scale, while on a large scale the magnetization rotates stochastically over the sample. The magnetization law on the approach to saturation is entirely determined by the random anisotropy effect, leading to certain predictions depending on the dimensionality of the system. ${ }^{29}$

For all the three samples, the best fit to the $M(H)$ data is provided by $1 / H$ law at low fields and by $1 / H^{2}$ law at high fields (see Fig. 8). Then, according to the theory, our RE-TM systems behave as two-dimensional amorphous ferromagnets. This is not surprising since the es-

TABLE I. Structure of the samples.

\begin{tabular}{lccccc}
\hline Sample & Substrate & $\begin{array}{c}\text { Buffer fundamental } \\
\text { sequence }(F, S)\end{array}$ & $\begin{array}{c}\text { Repetition } \\
\text { of } F, S\end{array}$ & RE/TM & $\begin{array}{c}\text { Total volume }^{\mathrm{b}} \\
\left(\mathrm{cm}^{3}\right)\end{array}$ \\
\hline $\mathrm{Fe} / \mathrm{Sm}$ & Kapton foil & $100 \AA \mathrm{Cu}[\mathrm{Fe}(3 \AA) / \mathrm{Sm}(2 \AA)]_{\times 6}$ & 15 & $\mathrm{Fe}_{4} \mathrm{Sm}$ & $7 \times 10^{-5}$ \\
$\mathrm{Fe} / \mathrm{Tb}$ & Kapton foil & $100 \AA \mathrm{Ag} \mathrm{Fe}(3 \AA) / \mathrm{Tb}(4 \AA)$ & 50 & $\mathrm{Fe}_{3} \mathrm{~Tb}$ & $5 \times 10^{-5}$ \\
$\mathrm{Co} / \mathrm{Sm}$ & Kapton foil & $100 \AA \mathrm{Cu}[\mathrm{Co}(4 \AA) / \mathrm{Sm}(3 \AA)]_{\times 5}$ & 11 & $\mathrm{Co}_{4} \mathrm{Sm}$ & $8 \times 10^{-4}$ \\
$\mathrm{Co}$ & Kapton foil & $100 \AA \mathrm{Cu}[\mathrm{Co}(30 \AA) / \mathrm{Cu}(100 \AA)]$ & 20 & & $6 \times 10^{-5}$ \\
\hline \hline
\end{tabular}

${ }^{a}$ The composition of the samples was obtained by calculating the evaporated mass of each metal, by using a crystal quartz oscillator. We have also performed the electronic microprobe analysis of each sample and obtained compositions in agreement with those calculated.

${ }^{\mathrm{b}}$ In the measurements we have used samples containing 40 films of each composition. 


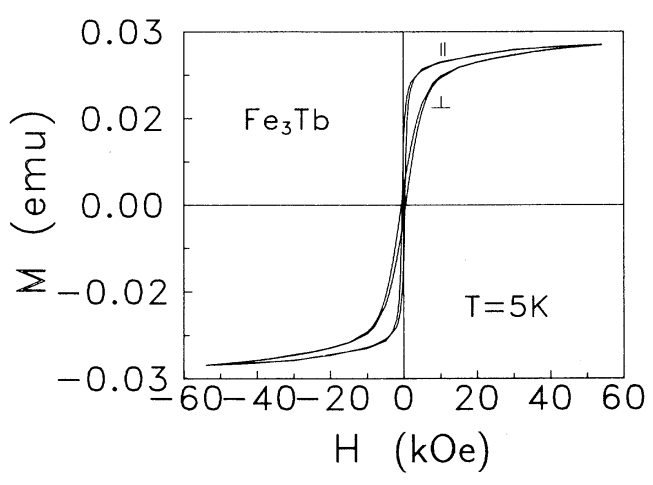

FIG. 6. Hysteresis loops at $5 \mathrm{~K}$ for the FeTb sample in parallel and perpendicular directions to the film plane.

timated ferromagnetic correlation length exceeds the thickness of the film for all samples.

\section{Low field measurements}

Zero-field-cooled (ZFC) and field-cooled (FC) measurements of $M(T)$ at low field were performed to obtain information about the irreversibility and freezing of magnetic moments. The remarkable feature of the ZFC curve is the existence of a broad peak in the $M(T)$ dependence. In Fig. 9 we show the ZFC and FC curves for the FeSm sample. This indicates the presence of the broad distribution of relaxation times for the metastable magnetic states. Most likely, these metastable states are related to the orientation of ferromagnetic clusters with respect to the applied field and the effective anisotropy axes. One cannot exclude also that defects and irregularities of the geometry of the film are responsible for some of the metastable states.

\section{Magnetic relaxation}

All samples show a pronounced long-time magnetic relaxation down to $1.8 \mathrm{~K}$, the lowest experimental temperature. The relaxation $\operatorname{law}$ is $\log (t)$ or very close to $\log (t)$. Figures 10 and 11 show the variation of $M$ as a function

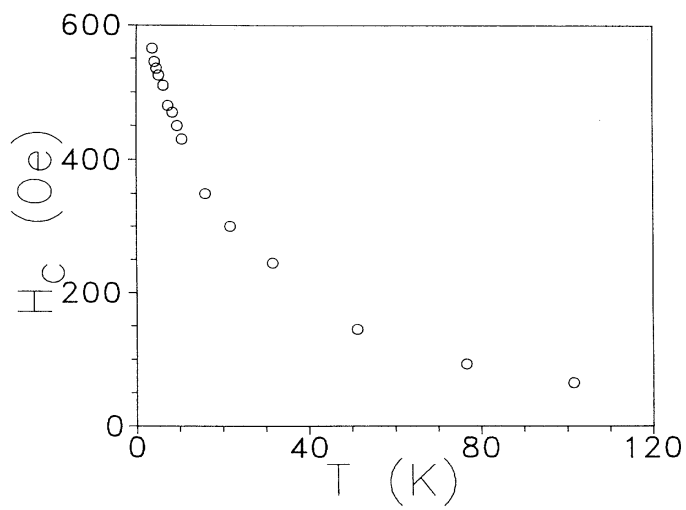

FIG. 7. Variation with temperature of the coercive field for the $\mathrm{Fe}_{4} \mathrm{Sm}$ sample.
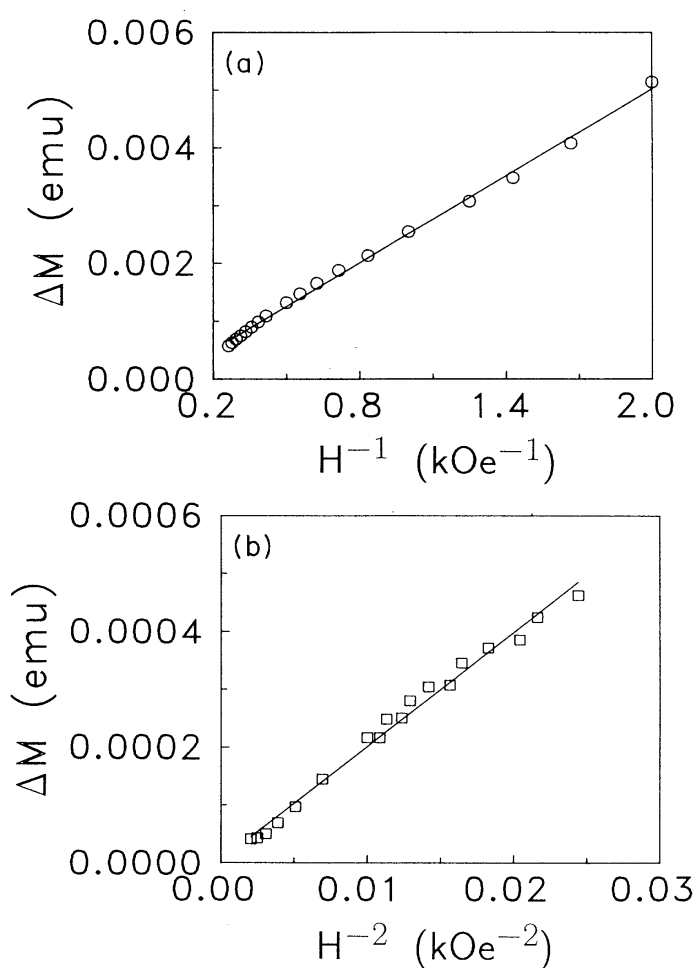

FIG. 8. Two regimes in approaching to saturation for the CoSm: (a) $\Delta M \propto 1 / H$. (b) $\Delta M \propto 1 / H^{2}$.

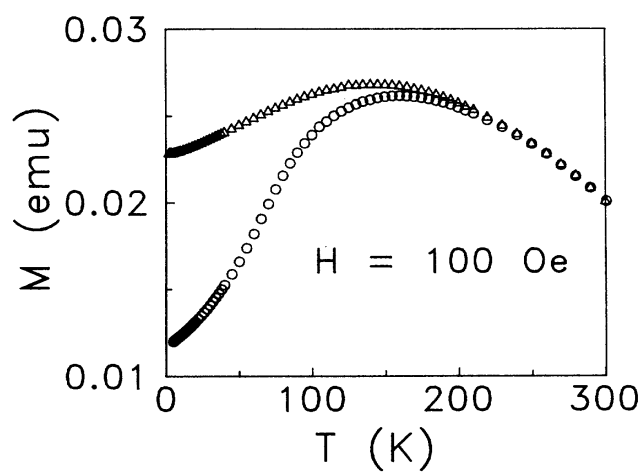

FIG. 9. Low field magnetization curves for FeSm obtained in a ZFC-FC process when $H=100 \mathrm{Oe}$ is applied parallel to the film plane.

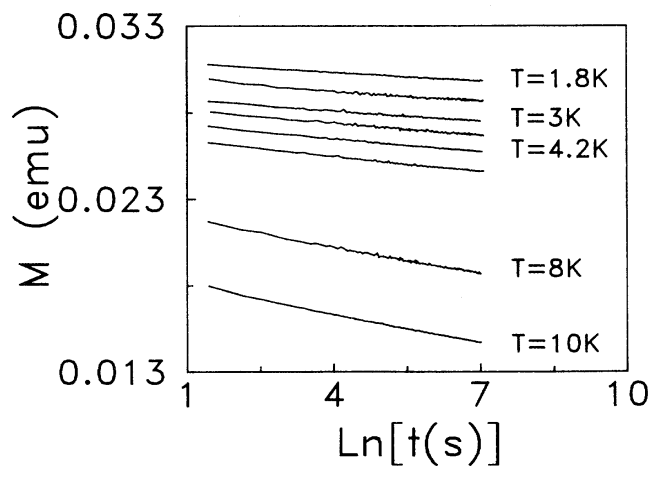

FIG. 10. Time dependence for the thermoremanence magnetization of $\mathrm{CoSm}$ at different temperatures, in the presence of -100 Oe. 


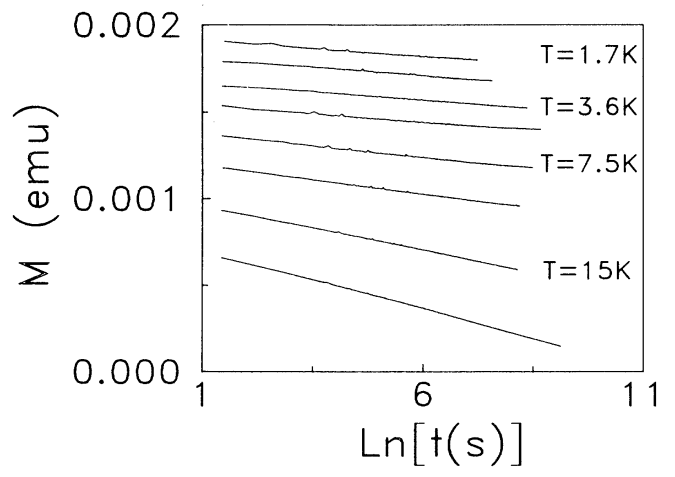

FIG. 11. Time dependence for the thermoremanence magnetization of the $\mathrm{FeTb}$ at different temperatures, in the presence of -100 Oe.

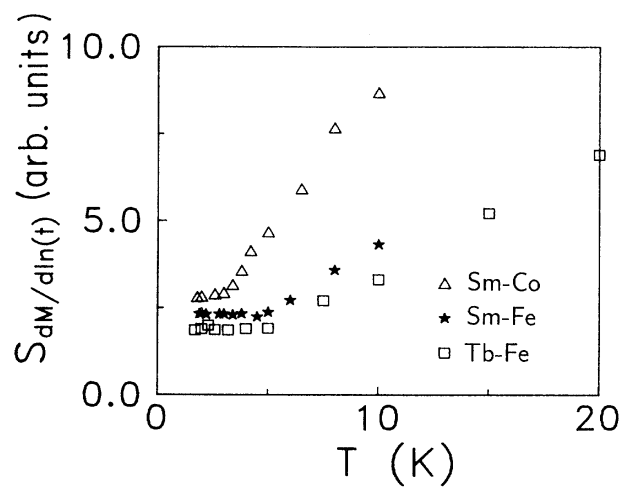

FIG. 12. Temperature dependence of the magnetic viscosity $S(T)$ for the three samples.

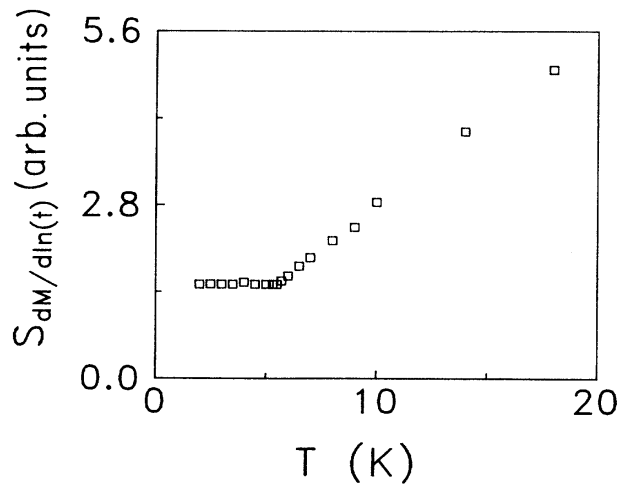

FIG. 13. Temperature dependence of the magnetic viscosity $S(T)$ for the FeTb sample. This curve is the same as that appearing in Fig. 12 for $\mathrm{FeTb}$ but with more experimental points in order to make clear the existence of the sharp transition.

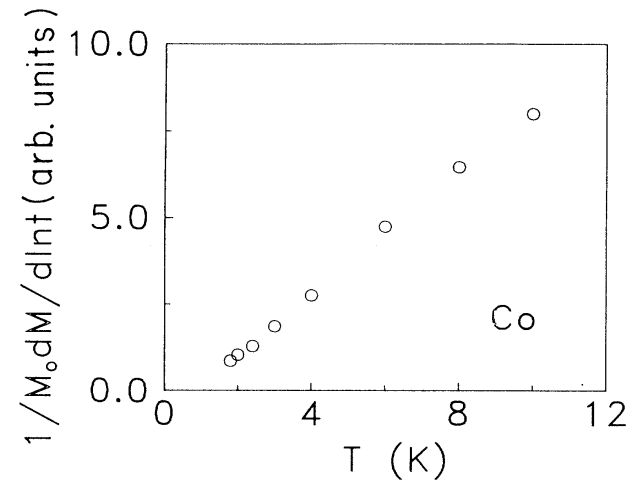

FIG. 14. Temperature dependence of the magnetic viscosity $S(T)$ for the Co thin film sample, which was used as a control sample.

of $\ln (t)$ at different temperatures for two samples. The temperature dependence of the magnetic viscosity, $S(T)$, defined by Eq. (28) is shown in Fig. 12. At high temperature, $S(T)$ depends linearly on $T$. This dependence extrapolates to zero at $T=0$, in accordance with the theory. Our central observation is that $S(T)$ is not dependent on temperature at low temperature. The existence of the plateau in $S(T)$, with a sharp transition from thermal to nonthermal relaxation, is obvious (see Fig. 13). It cannot be attributed to any experimental artifact, since in other systems we observed relaxation extrapolating to zero at $T=0$. In Fig. 14 we present the $S(T)$ dependence for a Co thin film of $30 \AA$ thickness (see Table I), which does not show the two regimes. This sample was used as a control sample in our relaxation experiments.

\section{DISCUSSION}

In Sec. II we presented a few mechanisms of tunneling and discussed possible temperature and time dependence of the magnetic relaxation. Let us summarize the main conclusions of the theory.

First, in all cases where barriers are created by the magnetic anisotropy, one should expect that $\mu_{B}\left(H_{\|} H_{\perp}\right)^{1 / 2}$ (with $H_{\|}$being the anisotropy responsible for the barrier and $H_{\perp}$ being the anisotropy field responsible for $[H, M] \neq 0$ ) gives the correct scale of the crossover temperature. This turns out to be true for such distinct processes as tunneling in single-domain particles, quantum nucleation of domains in bulk magnets, and quantum diffusion of domain walls. Field-dependent parameters, such as $\epsilon^{1 / 2}$ in Eq. (5) and $\left(h_{c} \epsilon\right)^{1 / 4}$ in Eq. (8) can change the above estimate only for very special experimental situations. Thus, in materials with high anisotropy, $H_{\|} \sim H_{\perp} \sim 1 \mathrm{~T}$, the observation of the crossover at a few kelvin would be quite natural.

Second, it has been demonstrated that in relaxationtype experiments a complex magnetic system is in a critical state which must follow the $\log (t)$ relaxation law given by Eq. (28). The coefficient in front of $\log (t)$ (magnetic viscosity) must be independent of temperature in the quantum relaxation regime, and must grow with tem- 
perature in the thermal regime.

Magnetic properties of our random magnets are quite well described by the random anisotropy model. ${ }^{29}$ According to this model, the ferromagnetic exchange averages on a small scale where the local magnetization is formed, while the direction of the magnetization wanders randomly, leading to a finite ferromagnetic correlation length. This state can be thought of as formed by small ferromagnetic clusters whose magnetization directions are separated by anisotropy barriers.

Let us list major features of the relaxation process, observed experimentally, and compare them with theoretical expectations.

(1) A small part of the total $M$ is relaxing.

(2) Relaxation follows the $\log (t)$ law.

(3) $S(T) \propto T$ at high $T$.

(4) $S(T)$ is independent of $T$ at low $T$.

(5) The crossover occurs at a few kelvin.

The first means that the decay of metastable states occurs in a small fraction of the volume. This is what one would expect looking at the ZFC $M(T)$ curve (Fig. 9). According to this curve, most of the metastable states freeze out below $T_{B} \sim 100 \mathrm{~K}$ because of large barrier heights. The broad maximum in Fig. 9 indicates, however, that some fraction of the volume remains in a superparamagnetic state down to very low temperatures, $T \ll T_{B}$. In other words, there are small clusters whose magnetic states are separated from the absolute energy minimum by barriers much lower than average. As follows from Eqs. (28) and (29), the relaxing fraction of the total magnetization, on the time scale of the experiment, must be of the order of $T^{*} / T_{B}$. This agrees with the experimental data.

The $\log (t)$ relaxation is what one should expect in a critical state, or for a complex system having a broad distribution of energy barriers. Such a relaxation is a simple reflection of the fact that metastable states, having lower barriers, decay on a shorter time. As the observation time is running, the system comes to higher and higher barriers which are more and more difficult to overcome. At any time of the observation $t$, metastable states, which are currently decaying, are characterized by the lifetime $\Gamma^{-1} \sim t$. This situation always lead to the $\log (t)$ relaxation, as has been demonstrated in Sec. II D.

The proportionality of $S(T)$ to $T$ at $T_{c} \ll T<<T_{B}$ is in accordance with the theoretical expectation, Eq. (29). It may also indicate weak correlations between magnetic clusters. The latter would be quite natural for our systems. First, the magnetic dipole interaction in these systems, which may be responsible for the interaction between the clusters, is much lower than the anisotropy energy responsible for the barriers. Secondly, since a small fraction of the total moment is relaxing, the clusters simultaneously involved in the relaxation process must be separated by a large distance, which should additionally decrease their interaction.
The existence of the plateau in the $S(T)$ dependence at low temperature is exactly what one would expect in the presence of quantum tunneling of magnetization, see Eq. (29) and Fig. 4. One may notice that the transition from thermal to quantum regime is rather sharp, certainly sharper than for macroscopic quantum tunneling observed in Josephson junctions. ${ }^{33}$ This is again in accordance with the theoretical expectation of weak dissipation in magnetic tunneling ${ }^{13,14,16}$ and the prediction of a sharp transition in systems with weak damping. ${ }^{22}$ It should be noted that, according to Eq. (29), the distribution in the geometrical shape (not the height) of the barriers, must smear the transition. The sharpness of the thermal-to-quantum crossover indicates, therefore, that most of the barriers have the same physical nature.

The numerical analysis of magnetization curves, based upon the random anisotropy model, ${ }^{29}$ shows that the anisotropy field in our samples is somewhat smaller but typically of the order of the effective exchange field. Correspondingly, ${ }^{29}$ the effective anisotropy field must be of the order of the local anisotropy, and the local magnetization must be well off the local easy direction. This gives $H_{\|} \sim H_{1} \sim H_{\text {an }}$ where $H_{\text {an }}$ is the average local anisotropy field. In all RE-TM systems studied, $H_{\text {an }}$ was greater than $1 \mathrm{~T}$. Consequently, $T_{c}$ of the order of a few kelvin is what one should expect in these materials.

Note that the observed crossover cannot be the crossover between two different thermal regimes (e.g., from coherent rotation of $M$ in small clusters to tunneling of domain walls) because the magnetic viscosity $S(T)$ for any thermal regime must be heading to zero as $T \rightarrow 0$.

One may try to invent a sophisticated model of interactions between clusters which would mimic the observed behavior of the magnetic viscosity. However, no such model is known yet. On the other hand, the entire correspondence between observations and theoretical conceptions on magnetic relaxation provides strong confidence that we are dealing with quantum tunneling of magnetization. Based upon quantum theory and conventional magnetism, the existence of this phenomenon below a few kelvin seems unavoidable. Relaxation experiments provide an ideal situation for the observation of this effect. They automatically drive the system into a state with extremely low-energy barriers, where the nonthermal, subbarrier "leaking" of the magnetization from metastable states occurs. A rigorous statistical theory of this effect has yet to be developed, as the observation of a single tunneling event remains a challenging experimental task.

\section{ACKNOWLEDGMENTS}

The work of E.C. was supported by NSF Grant No. DMR-9024250. X.X.Z. thanks the Spanish "Ministerio de Educación y Ciencia" for financial support. J.T. thanks the CICYT for financial support. 
${ }^{1}$ E. M. Chudnovsky, Zh. Eksp. Teor. Fiz. 77, 2157 (1979) [Sov. Phys. JETP 50, 1035 (1979)].

${ }^{2}$ E. M. Chudnovsky and L. Gunther, Phys. Rev. Lett. 60, 661 (1988); Phys. Rev. B 37, 9455 (1988).

${ }^{3}$ For a recent review see, e.g., P. C. E. Stamp, E. M. Chudnovsky, and B. Barbara, Int. J. Mod. Phys. B 6, 1355 (1992).

${ }^{4}$ X. X. Zhang, Ll. Balcells, J. M. Ruiz, O. Iglesias, J. Tejada, and B. Barbara, Phys. Lett. A 163, 130 (1992); X. X. Zhang, Ll. Balcells, J. M. Ruiz, J. L. Tholence, B. Barbara, and J. Tejada, J. Phys. Condens. Matter 4, L163 (1992); Ll. Balcells, X. X. Zhang, F. Badia, J. M. Ruiz, C. Ferrater, and J. Tejada, J. Magn. Magn. Mater. 109, L159 (1992); J. Tejada, Ll. Balcells, and X. X. Zhang, ibid. 118, 65 (1993); Ll. Balcells, J. L. Thoulence, S. Linderoth, B. Barbara, and J. Tejada, Z. Phys. B 89, 209 (1992); J. Tejada, X. X. Zhang, and B. Barbara, J. Magn. Magn. Mater. (unpublished).

${ }^{5}$ C. Paulsen et al., Phys. Lett. A 161, 319 (1991), and references therein; X. X. Zhang, R. Zquiak, J. Tejada, and B. Barbara, J. Phys. Condens. Matter 4, 10347 (1992).

${ }^{6}$ D. D. Awschalom et al., Phys. Rev. Lett. 65, 783 (1990); 68 3092 (1992).

${ }^{7}$ The only exemption from that rule would be the case of a pure uniaxial anisotropy, e.g., along the $Z$ axis, which commutes with $M_{z}$. In this case the commutation will be violated by the magnetic field applied at some angle to the $Z$ axis.

${ }^{8}$ See, e.g., M. Tinkham, Introduction to Superconductivity (McGraw-Hill, New York, 1975) for details, and Refs. 9-11 for recent experiments.

${ }^{9}$ Y. Yeshrum and A. P. Malozemoff, Phys. Rev. Lett. 60, 2202 (1988).

${ }^{10}$ Y. Xu et al., Phys. Rev. B 40, 10882 (1989).

${ }^{11}$ L. Fruchter et al., Phys. Rev. B 43, 8709 (1991).

${ }^{12}$ C. P. Bean, Phys. Rev. Lett. 8, 250 (1962).

${ }^{13}$ P. C. E. Stamp, Phys. Rev. Lett. 66, 2802 (1991).

${ }^{14}$ E. M. Chudnovsky, O. Iglessias, and P. C. E. Stamp, Phys.
Rev. B 46, 5392 (1992).

${ }^{15}$ A. O. Caldeira and A. J. Leggett, Phys. Rev. Lett. 46, 211 (1981); Ann. Phys. (N.Y.) 149, 374 (1983).

${ }^{16}$ A. Garg and G. H. Kim, Phys. Rev. Lett. 63, 2512 (1989); Phys. Rev. B 43, 712 (1991).

${ }^{17}$ I. Affleck, Phys. Rev. Lett. 46, 388 (1981).

${ }^{18}$ A. I. Larkin and Yu. N. Ovchinrikov, Pis'ma Zh. Eksp. Teor. Fiz. 37, 322 (1983) [JETP Lett. 37, 382 (1983)].

${ }^{19}$ H. Grabert and U. Weiss, Phys. Rev. Lett. 53, 1787 (1984).

${ }^{20}$ C. Scharf, W. F. Wreszinski, and J. L. van Hemmen, J. Phys. A 20, 4309 (1987).

${ }^{21}$ E. M. Chudnovsky, Phys. Rev. A 46, 8011 (1992).

${ }^{22}$ H. Grabert, P. Olschowski, and U. Weiss, Phys. Rev. B 32, 3348 (1985).

${ }^{23}$ W. Zwerger, Phys. Rev. A 31, 1745 (1985).

${ }^{24}$ P. S. Riseborough, P. Hänggi, and E. Freidkin, Phys. Rev. A 32, 489 (1985).

${ }^{25}$ P. W. Anderson and Y. B. Kim, Rev. Mod. Phys. 36, 39 (1964).

${ }^{26}$ See also D. K. Lottis, R. M. White, and E. D. Dahlberg, Phys. Rev. Lett. 67, 362 (1991), where a similar approach is developed for ferromagnets.

${ }^{27}$ R. Street and J. C. Wooley, Proc. Phys. Soc. London, Sect. A 62, 562 (1949).

${ }^{28}$ M. Uehara and B. Barbara, J. Phys. (Paris) 47, 235 (1986), and references therein.

${ }^{29}$ E. M. Chudnovsky and R. A. Serota, Phys. Rev. B 26, 2697 (1982); E. M. Chudnovsky, W. M. Saslow, and R. A. Serota, ibid. 33, 251 (1986); see also E. M. Chudnovsky, J. Appl. Phys. 64, 5770 (1988) for a review and Refs. 30-32 for most recent experimental works.

30J. Tejada et al., Phys. Rev. B 41, 858 (1990); 44, 7698 (1991).

${ }^{31}$ P. M. Gerring et al., Phys. Rev. B 41, 9134 (1990).

${ }^{32}$ B. Barbara et al., J. Phys. I (Paris) 2, 101 (1992).

${ }^{33}$ J. Clark et al., Science 239, 992 (1988), and references therein. 